\title{
The evidence for endorsing the use of short dental implants remains inconclusive
}

\author{
Abstracted from \\ Telleman G, Raghoebar GM, Vissink A, den Hartog L, Huddleston Slater JJ, Meijer HJ. \\ A systematic review of the prognosis of short $(<10 \mathrm{~mm})$ dental implants placed in the \\ partially edentulous patient. / Clin Periodontol 2011; 38: 667-676. \\ doi: 10.1111/j.1600-051X.2011.01736.x. Epub 2011 May 12. \\ Address for correspondence: Gerdien Telleman. Department of Oral and Maxillofacial Surgery, \\ University Medical Center Groningen and University of Groningen, Groningen, The Netherlands. \\ E-mail: g.telleman@kchir.umcg.nl
}

\section{Question: What is the prognosis of short implants when placed in the partially edentulous patient?}

Data sources Medline and Embase databases were searched between 1980 and October 2009 using free text and MeSH terms. Reference lists of retrieved publications were checked.

Study selection Randomised-controlled trials or prospective cohort studies were eligible that included partially edentulous patients in whom at least five implants (each of length $<10 \mathrm{~mm}$ ) were placed and where follow up was $>1$ year. Main outcomes were estimated failure rate per year and the estimated implant survival rate after two years. Failure was defined as implant removal due to loss of integration, implant mobility, symptoms as pain, neuropathies, paraesthesia, violation of the mandibular canal or psychological reason. Studies designated as 'methodologically unacceptable' were excluded. This was based on the validity assessment described below.

Data extraction and synthesis One reviewer extracted data from all included trials and another extracted from $25 \%$ of them. Validity of included studies was assessed using 'quality assessment of a cohort study' and 'quality assessment of a randomised clinical trial' developed by the Dutch Cochrane Centre. Authors were contacted by email when there were missing data. Sources of heterogeneity were explored using stratified analyses for the determinants surface topography, location (maxilla versus mandible), smoking and bone augmentation procedures. Results Twenty-nine studies were eligible including 2611 short implants (lengths 5, 6, 7, 8, 8.5, 9 and $9.5 \mathrm{~mm}$ ). Twenty-eight were prospective cohort studies and one was a randomised controlled trial. The authors do not present a summary of the quality of the studies. The mean follow up was 3.7 years (range 1.6 - 8.1 years). There was mild to moderate heterogeneity between the studies depending on the length of the implants placed. The estimated survival rate after two years ranged from $93.1 \%$ (95\% Cl: 79.7-100\%) for 5mm implants to $98.6 \%$ (95\% Cl: $94.6-100 \%$ ) for $9.5 \mathrm{~mm}$ implants.

Conclusions There is growing evidence that placement of short $(<10 \mathrm{~mm})$ implants can be successful in the partially edentulous patient.

\section{Commentary}

About a decade ago the FDI World Dental Federation initiated a project with the objective to critically appraise the scientific evidence for claims of relationship between characteristics of root formed dental implants and clinical performance. ${ }^{1}$ In the exhaustive report the authors addressed the prevailing opinion at the time amongst researchers and clinicians that the short implants failed more frequently than the longer implants. Following a critical appraisal of the underlying evidence for this prevailing opinion it was cautioned that: '... any demonstrable numerical relationship between two clinical variables in an often extensive and heterogeneous data set may in theory also be due to confounding clinical or patient factors, or it can be just a spurious statistical phenomenon. A prospective study that addresses the influence of implant length on treatment success, preferably randomised and/or blinded, can provide indications as to the extent to which this may be an aetiological factor for implant failure. As no such studies have been carried out, it cannot be ruled out that the reported association between implant lengths and clinical failure is a reflection of anatomical limitations in actual treatment situations. In other words, implant length is a surrogate variable for what actually represents differences in case and site selections in clinical trials.'

Since the 2003 FDI report, six systematic reviews (SRs) have been published about short implants and outcomes, ${ }^{2-7}$ supplementing the excellent review paper by Telleman and co-workers. ${ }^{8}$ Unfortunately, the statement above still holds true in 2011. Not a single study amongst the approximately 150 papers to which the seven SRs refer has been specifically designed to appraise the effects of implant lengths on clinically relevant outcomes. As a consequence, clinicians and investigators are left to struggle with identifying and interpreting any kind of clinical data that contain some element of information about the possible effects of implant length on treatment outcomes. Hence, the potential for bias and opinions based on flawed deductive reasoning is high.

It is unclear why many clinicians routinely use the maximum implant length achievable within the relevant anatomical limitations. Admittedly, laboratory bench-studies have shown that higher pull-out forces may be required for longer implants, but the clinical relevance of such tests is unclear. Two- and three-dimensional photo-elastic studies, finite element analysis and other statistical modeling methods have demonstrated how various levels of compressive and tensile stresses can develop within the implant, the implant:tissue interface and/or in the cortical and trabecular bone. However, these laboratory experiments have failed to elucidate 
Table 1. Systematic reviews $\mathbf{2 0}^{\mathbf{2 - 8}}$ reporting whether implant length is associated with clinical performance (Yes / No) statistically adjusted or corrected (Yes/No/Not analysed) for clinical variables likely to be considered as study confounders (Discussed)

\begin{tabular}{|c|c|c|c|c|}
\hline & Yes & No & Not analysed & Discussed \\
\hline Implant Length & $2^{*}, 3,4^{*}, 7^{*}, 8^{*}$ & $2^{*}, 5^{*}, 6^{*}, 7^{*}, 8^{*}$ & & \\
\hline Smoking & 8 & 6 & $2,3,4,5^{*}, 7$ & \\
\hline Concurrent Periodontitis & & & $2,3,4,5^{\star}, 6,7,8$ & \\
\hline Bone Quality (index) & & & $2,3,4,5,7,8$ & 3,4 \\
\hline Clinician training/skills & & & $2,3,4,5,6,7,8$ & 4 \\
\hline Surgical technique & & & $2,3,4,5,6,7$ & 4 \\
\hline Bone augmentation & & 6,8 & $2,3,4,5,7^{*}$ & \\
\hline Implant Surface & $2 *, 7$ & $2 *, 6,8$ & $3^{*}, 4,5^{*}$ & 4 \\
\hline Implant diameter & & $4 *, 6,7$ & $2,5,8$ & $3^{*}$ \\
\hline Implant tapered vs straight & & & $2,3,4,5,6,7,8$ & \\
\hline Mandible vs Maxilla & $2^{*}, 7^{*}, 8$ & $2^{*}, 6$ & $3,4,5$ & 3 \\
\hline Anterior vs Posterior & $7^{*}$ & 6 & $2,3,4,5,8$ & 3 \\
\hline Partial dentate vs edentulous & & $5^{\star}, 6,7$ & $2^{*}, 3,4,8^{*}$ & \\
\hline 1 vs 2 stage surgery & & 6 & $2,3,4,5,7,8$ & \\
\hline Primary stability (e.g., ISQ) & & & $2,3,4,5,6,7,8$ & \\
\hline Immediate loading & & 6 & $2,3^{*}, 4,5,7,8$ & \\
\hline Fixed vs Single & & 6,7 & $2,3,4,5,8$ & \\
\hline Fixed versus removable & & 6,7 & $2,3,4,5$ & \\
\hline Fixed FDP with cantilever & & & $2,3,4,5,6,7,8^{*}$ & \\
\hline Study methodology & & 6 & $2,3,4,5,7^{*}, 8^{*}$ & 7 \\
\hline Follow-up time & $6^{*}$ & & $2,3,4,5,7,8^{*}$ & $4^{*}, 6$ \\
\hline Author conflict of interest & & & $2,3,4,5,6,7,8$ & 6 \\
\hline \multicolumn{5}{|c|}{ 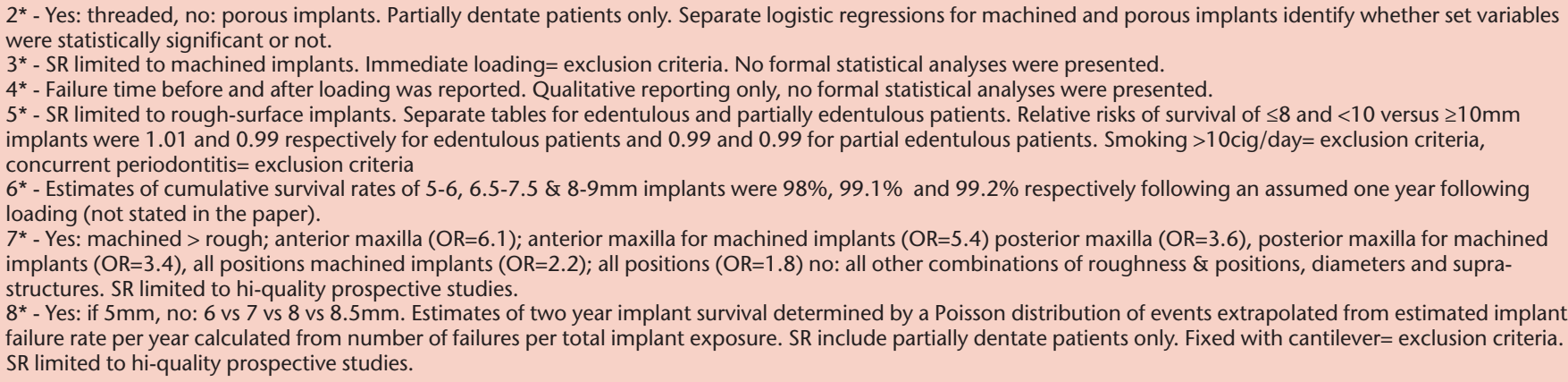 } \\
\hline
\end{tabular}

the relationship between occlusal load and the dissipation of forces to the implant:tissue interface in vivo, and whether the stresses achieved are actually detrimental remains elusive from both an internal and external study validity perspective. We simply don't know what is too little or too much stress; only carefully controlled clinical studies in humans can determine this.

Two different primary questions are being addressed in the cited SRs: ${ }^{1-8}$ 'What is the clinical performance of implants shorter than, e.g, $\leq 7 \mathrm{~mm}{ }^{2}$ or $\leq 9 \mathrm{~mm} ?^{\prime \prime 6,8}$ and, 'Do the longer implants demonstrate better clinical performance than the shorter ones, eg, $10 \mathrm{~mm}$ vs $8.5 \mathrm{~mm}$ vs $7 \mathrm{~mm} ;{ }^{3} 10,11.5$ or $13 \mathrm{~mm}$ vs $8.5,8,7$ or $6 \mathrm{~mm} ;{ }^{4} \geq 10 \mathrm{~mm}$ vs $<10$ vs $\leq 8 \mathrm{~mm} ;^{5}$ or $\geq 10 \mathrm{~mm} v \mathrm{~s} \geq 7$ and $<10 \mathrm{~mm} ?^{7}$ The different questions are reflected by the choice and selection of primary studies used for extracting data to answer the questions. While the first question is about prognosis the second is about making comparisons between two or more different interventions. Some will suggest that comparing implant dimensions (including length) may be of interest, while a contrarian opinion will be that it is more relevant to compare the use of short implants versus some form of bone augmentation intervention made necessary by the placement of a longer implant.

Also, the time element that has been considered in the various SRs differs. The three prognosis SRs aim to address: What is the clinical performance of the $\leq 7 \mathrm{~mm}$ implants over a minimum of 2 years $^{2}$ or $\leq 9 \mathrm{~mm}$ over a minimum of 1 year?6,8 The comparison SRs seek to answer: Do the longer implants demonstrate better clinical performance than 
the shorter ones in general, ${ }^{4}$ alternatively over a minimum of 1 year, ${ }^{3,5}$ alternatively within the first year of loading. ${ }^{7}$

The cited SRs ${ }^{1-8}$ have extracted data from between $12^{2}$ and $54^{6}$ primary studies, and the authors have used different strategies to attempt to quality assure the search for the primary research studies. Some limit the search of bibliographic databases to Medline $e^{2-4,6}$ while others include also Embase and/or the Cochrane library. $1,5,7,8$ Five SRs include data from all study designs ${ }^{1-4,6}$ while three have limited their data extraction to prospective ${ }^{5}$ or even 'high quality'/'methodologically acceptable ${ }^{7,8}$ prospective studies. Two SRs limit their focus to partially dentate patients, ${ }^{2,8}$ while others are limited to only 'Brånemark-compatible ${ }^{3}$ or 'rough-surface' implants. ${ }^{5}$ Moreover, the level of sophistication of the statistical analyses range from applying multivariate logistic regression ${ }^{2}$ or Mantel-HaenzelTests $^{7}$ to the purely descriptive. ${ }^{1}$ Outcomes that are analysed are either crude failure rates ignoring the observation time element, $, 3,3,5,7$ cumulative survival rates (CSRs), ${ }^{4,6}$ or as failures per implant exposure time as a basis for estimates of two-year CSR based on a Poisson distribution of adverse events. ${ }^{8}$ Meta-analyses were applied in one SR to estimate the relative risks of selecting shorter implants ${ }^{5}$ while another estimated CSRs as a function of several clinical variables, including implant lengths. ${ }^{6}$ Statistical checks for publication bias was reported in two SRs, ${ }^{5,7}$ while tests for statistical heterogeneity amongst the primary studies was applied in four SRs. ${ }^{5-8}$

The introductory quote from the 2003 FDI report ${ }^{1}$ stated that '... any demonstrable numerical relationship between two clinical variables in an often extensive and heterogeneous data set may in theory also be due to confounding clinical or patient factors...'. A striking lack of detail regarding these factors in the primary reports is discussed by all eight SR author groups. ${ }^{1-8}$ However, while some author groups chose to simply exclude potentially eligible articles due to lack of information in the reports, other groups made attempts to collect, with more or less success, additional information about methodological content from the original authors of the primary studies. $5,7,8$ The quality of a SR with or without a meta-analysis can never be better than the quality of the primary studies; bluntly stated, this means 'garbage in, garbage out'. This fact may be the explanation for why some of the SRs following statistical analyses identify some potential confounding clinical co-variables and some do not (Table 1).
It is indeed a great challenge to interpret data-sets from methodologically weak clinical studies. Heterogeneous data sets add an additional level of confounding. Under these circumstances it takes courage by an independent clinician or researcher to express an opinion based on the available evidence. It is commendable that most of the authors of the reviews discuss their concerns about internal and external validity of the primary studies. The conclusions of the SRs clearly contrast, and presently it is unknown which one should be regarded as the most trustworthy.

So what clinical practice recommendations can be drawn from the current body of research? No strong recommendations, unfortunately. Short implants seem to be a viable alternative to longer implants as well as to augmentation techniques over a follow-up of about two years. Beyond the first years following implant placement the current scientific data are insufficient for providing clinical guidance. Moreover, no strong prognostic factors associated with an adverse outcome of using short dental implants have been identified - except perhaps as Telleman and co-workers have identified, ${ }^{8}$ ie, a likely less predictable positive outcome in the maxilla amongst heavy smokers.

Asbjorn Jokstad

University of Toronto Faculty of Dentistry, Toronto, Canada

1. Jokstad A, Braegger U, Brunski JB, Carr AB, Naert I, Wennerberg A. Quality of Dental Implants. Int Dent / 2003; 53 Suppl 2: 409-433.

2. Hagi D, Deporter DA, Pilliar RM, Arenovich T. A targeted review of study outcomes with short $(<$ or $=7 \mathrm{~mm}$ ) endosseous dental implants placed in partially edentulous patients. J Periodontol 2004; 75: 798-804.

3. das Neves FD, Fones D, Bernardes SR, do Prado CJ, Neto AJ. Short implants - an analysis of longitudinal studies. Int / Oral Maxillofac Implants 2006; 21: 86-93.

4. Renouard F, Nisand D. Impact of implant length and diameter on survival rates. Clin Oral Implants Res 2006; 17 Suppl 2: 35-51.

5. Kotsovilis S, Fourmousis I, Karoussis IK, Bamia C. A systematic review and meta-analysis on the effect of implant length on the survival of rough-surface dental implants. / Periodontol 2009; 80: 1700-1718.

6. Annibali S, Cristalli MP, Dell'aquila D, Bignozzi I, La Monaca G, Pilloni A. Short Dental Implants: A Systematic Review. J Dent Res 2011 Oct 27. Online Pre-publication.

7. Pommer B, Frantal S, Willer I, Posch M, Watzek G, Tepper G. Impact of dental implant length on early failure rates: a meta-analysis of observational studies. J Clin Periodontol 2011; 38: 856-863.

8. Telleman G, Raghoebar GM, Vissink A, den Hartog L, Huddleston Slater JJ, Meijer HJ. A systematic review of the prognosis of short $(<10 \mathrm{~mm})$ dental implants placed in the partially edentulous patient. / Clin Periodontol 2011; 38: 667-676.

Evidence-Based Dentistry (2011) 12, 99-101. doi:10.1038/sj.ebd.6400821 